\title{
The role of accessibility and usability in bridging the digital divide for students with disabilities in an e-learning environment
}

\author{
M. J. Maboe \\ School of Computing, University of \\ South Africa \\ Florida, South Africa \\ maboemj@UNISA.ac.za
}

\author{
M. Eloff \\ Institute for Corporate Citizenship, \\ University of South Africa \\ Pretoria, South Africa \\ eloffmm@UNISA.ac.za
}

\author{
M. Schoeman \\ School of Computing, University of \\ South Africa \\ Florida, South Africa \\ schoema@UNISA.ac.za
}

\begin{abstract}
To allow access to educational information for all people, including those with disabilities, the Internet and websites should be accessible and usable. Websites should provide timely and precise information effectively, efficiently and satisfactorily. Accessible and usable websites will create the necessary platforms for students to learn at open distance e-learning (ODeL) institutions without hindrances. Academic activities, such as accessing study material or contacting lecturers and other teaching and learning processes, are difficult to perform at an open distance learning institution without the use of computers and the Internet. This article investigates and reports on usability principles not satisfied based on time taken by students with disabilities to complete certain tasks, using the University of South Africa (Unisa) website and myUnisa, its e-learning website. The findings of the study indicate that not all usability principles for participants or students with disabilities are satisfied by this institution's e-learning websites. The study con-firms that welldeveloped e-learning platforms must guarantee efficient, effective and satisfactory use by all students, including those with disabilities. The study suggests that it is important for all the relevant stakeholders to be involved in the development of websites to ensure that accessibility and usability are properly adhered to. The aim is to reduce digital and social divides and the marginalisation of students, specifically when the target user groups have disabilities or limited capabilities.
\end{abstract}

\section{CCS CONCEPTS}

- Human-centered computing $\rightarrow$ Human-computer interaction; Universal usability; universal access and design; usability; interviews; • Usability laboratory $\rightarrow$ Usability testing

\section{KEYWORDS}

Universal access and design; usability; disabilities; ICT; elearning

\section{ACM Reference format:}

(C) [M.J. Maboe, M. Eloff, and M. Schoeman] [2018]. This is the authors' version of the work. It is posted here for your personal use. Not for redistribution. The definitive version was published In Proceedings of SAICSIT 2018, Port Elizabeth, South Africa, September 26-28, 2018, 7 pages, https://doi.org/10.1145//3278681.3278708

\section{INTRODUCTION}

Websites, including those of general higher education institutions and e-learning websites, should be accessible and usable to all people, including those with disabilities. In this way, learning through websites will be accessible to everyone [1]. Flexible websites will accommodate each user's needs, allowing people to access and use them properly.

This study was conducted at one of the largest ODeL institutions, the University of South Africa (Unisa). It is important for people with disabilities to be able to browse the Unisa and myUnisa websites with ease and satisfaction, and to achieve their intended goals while visiting these websites [1]. People with disabilities should be able to accomplish their planned tasks when they work on websites. They should not have to put in more effort in order to attain these results; that is, they should be able to achieve their anticipated results in a similar way that other people attain results when visiting websites.

If the means with which computers and physical spaces are accessed are not suitably designed, people with and even those without disabilities may experience difficulties in using computers, for example in reading text and/or hypertext on computer. They may also experience navigational problems and 
complications with images, photographs, diagrams, charts, interaction and animation, and video and audio within computing facilities [2]. Having a disability should not hamper a student's accessibility to and use of websites such as those of Unisa and its e-learning website, myUnisa. This is particularly important at an ODeL institution where students generally have to study online.

The different types of disabilities that are commonly found include mobility or ambulatory, visual, auditory, cognitive and neural disabilities [3]. This study investigated the impact of mobility, specific limited hand functioning, visual and auditory disabilities, on logging on and retrieving information from the elearning website of Unisa.

Given these types of disabilities, the following three research objectives were identified:

1. To examine how time of task completion relates to the usability principles;

2. To observe the principles which are problematic when participants try to accomplish specific tasks on Unisa's e-learning website; and

3. To recommend ways to improve efficiency and effectiveness of e-learning websites at this ODeL institution.

The paper is arranged as follows: the next section deals with universal access and the design and usability of the Unisa and myUnisa websites. The methodology adopted for the study and the findings of the study are then presented and discussed. The paper concludes with an argument for the integration of e-learning that caters for the needs of students with disabilities.

\section{UNIVERSAL USABILITY}

According to Horton and Leventhal [4] and Lidwell, Holden and Butler [5], universal usability entails, first of all, accessibility or universal access and design and, furthermore, usability.

\subsection{Universal access and design}

Accessibility or universal access and design can be logical or physical. Logical accessibility (for people with disabilities) ascertains the ease with which people with disabilities are, for example, able to read material; physical accessibility refers to physical accommodation such as access ramps or Braille in lifts, allowing people with disabilities to access their desired location with no or few limitations [6]. This highlights the fact that people with disabilities should be able to gain entry to physical facilities, all modes of transport and computer facilities in all locations [7]. Accessibility should be provided in such a way that there is access to physical spaces and access to learning material [8].

Universal design in learning (UDL) encourages access to learning material [7]. In this paper, we discuss UDL principles, as defined by the Center for Applied Special Technology [8-9].

Principle 1: Offer multiple means of representation

Checkpoint 1.1: Offer options for perception

Information should be understandable to all, based on their sensory perceptions with alternatives for the different formats (visual, auditory, text). Information should be adjustable so that it can meet each student's needs.

Checkpoint 1.2: Offer options for language, calculations, expressions and signs

Options should be provided to clarify text, mathematical expressions, symbols, syntax and structure to prevent misunderstanding of concepts.

Checkpoint 1.3: Provide options for comprehension

Enhance comprehension by linking new knowledge to previous understanding, emphasising important information, scaffolding knowledge and using models and feedback to aid comprehension and assist with knowledge transfer.

Principle 2: Offer multiple means of action and expression

Checkpoint 2.1: Offer choices for physical action

Hard-copy materials should not be the only means of support for students with disabilities; electronic documents or voice recordings should also be included. The curriculum should provide faultless interaction where universal technologies are used by students with disabilities to find their way in interacting with the curriculum.

Checkpoint 2.2: Offer options for expression and communication Allow alternative means of expression via multiple media to cater for learners with varying abilities and to develop learners' skills. Reduce scaffolding gradually to increase independence.

Checkpoint 2.3: Offer options for executive functions

Provide opportunities and assistance for goal-setting, planning and strategy development, managing information and resources and allow monitoring of progress.

Principle 3: Offer multiple means of engagement

Checkpoint 3.1: Provide options for engaging interest

Attract and retain attention by providing choices of how learning can be achieved, own goals set and progress towards goals monitored. Link learning to meaning experiences and provide a safe learning space.

Checkpoint 3.2: Provide options to support effort and persistence Offer possibilities for supporting effort and perseverance. For example, remind learners of their goals, provide a range of challenges with varying levels of difficulty, encourage cooperative learning and give encouraging feedback.

Checkpoint 3.3: Provide options for self-regulation

Encourage learners to set personal goals and support them in developing personal coping skills and self-assessment to maximise motivation.

Accessibility is expressed as the usability of a product, service or setting by people with the widest scope of capabilities. This means that accessibility is basically usability for the extreme potential set of identified users assisted [10]. This implies that no website can be usable without first being accessible. It is also important to note that accessibility and usability of websites are what is evaluated, not the people who use them. If UDL principles are followed properly, that will lead to usable websites which all students are able to use easily. 


\subsection{Usability, usability testing and eye tracking}

If a person cannot access a website, then there is no way for that person to determine how supportive the website is. While accessibility is one component of universal usability, usability is the other crucial part [11]. Usability is the simplicity with which a user is able to interact with their surroundings, for instance websites, regardless of obstacles [12]. The user's insight is central to the value of websites and will help users to see and understand the environment and what is essential to them [13].

Bevan, Carter and Harker [14] identify three aspects of usability:

"The extent to which a product can be used by specified users to achieve specified goals with effectiveness, efficiency, and satisfaction in a specified context of use." These aspects of usability can be explained as follows:

- Effectiveness is the ability of a user to positively use a website to discover information and complete tasks.

- Efficiency is a productive way to accomplish a goal after understanding it.

- Satisfaction is the sense of achievement after doing something successful.

In addition to efficiency and satisfaction, Nielsen [15] includes further attributes of usability:

- Memorability refers to the ability of the users to remember steps last taken in the system.

- Errors are the number of mistakes per user, how serious these mistakes are and the way users are able to resolve them.

- Learnability is the simplicity with which a person is able to solve a problem the first time.

These attributes are measurable and show how usable the application is [15].

Furthermore, Sauro and Kindlund [16] explain the quantitative model of usability as follows:

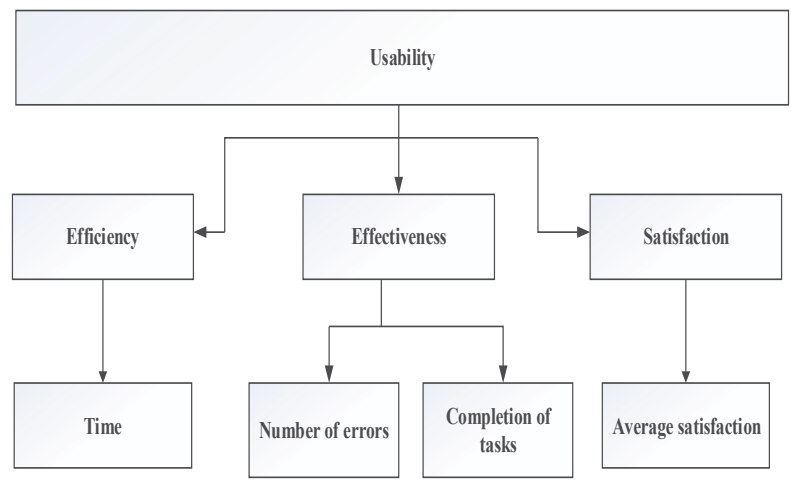

Figure 1: Quantitative model of usability

As seen in Fig. 1, the quantitative model of usability shows that tasks must be done within a short or suitable period and with few or no errors to a user's average satisfaction.

Usability testing determines whether the users are satisfied during their interaction with websites $[12,17]$. It aims to ensure that websites are accessible and usable to their intended audience, and the intention is not to measure users' abilities [12]. During the inception of usability testing in the 1980s, users' profiles were changed to include all users, including those with disabilities [18].

The design principles for usability used in this study include, but are not limited to, Budd's principles for modern Web applications [19], design principles by Dix, Finlay, Abowd and Beale [20], Mayhew's general principles of user interfaces [21], Molich and Nielsen's heuristics [22], design principles by Norman [23], Petrie and Power's evidence-based heuristics [23], Preece, Rogers and Sharp's principles on usability [12], Petrie and Power's evidence-based heuristics [24], Shneiderman and Plaisant's principles for design interfaces [25] and Tognazzini's basic principles of interface design [26]. These usability principles include:

- Accomplishing tasks, which means that websites should allow for actions that follow one another sequentially, up to the completion of the tasks;

- Consistency, which ensures that the systems behave in a similar manner for similar applications;

- Efficiency, which ensures that users' interaction with websites is supported promptly in meeting their needs;

- Familiarity, which refers to the extent to which participants relate their previous understanding to the actual computer domains when they use new applications;

- Flexibility, which refers to the means by which websites allow people with disabilities to accomplish their tasks in different ways;

- A help facility, which enables people to correct their mistakes by undoing or recovering from mistakes within a short space of time;

- Learnability, which is the extent to which websites make it easy for users to learn to use them;

- Recoverability, meaning that users' interaction with websites should be such that if users have made mistakes, they are able to notice them and recover to their initial state;

- Responsiveness, which is a situation in which system interruption of communication is minimised;

- Robustness, which is when websites recover from mistakes made by users in such a way that these mistakes do not affect the users of the websites;

- Simplicity, which allows users to work with a website so that it will be easy for them to interact with this website at any stage;

- Visibility, meaning that a website has visible functions and controls on what the users must do.

Dumas and Fox [18] identify the features of usability testing, all of which must be satisfied if a website is to be successful:

- The focus must be on the usability of websites.

- The actual users of websites are the participants. Participants in the evaluation of websites should be the real users of the websites. For this study, these were students with disabilities.

- Data is recorded for later analysis of participants' interviews. 
- Test results are communicated to stakeholders, in this case the students with disabilities.

Eye tracking monitors and records the movement of the participants' eyes on the computer screen instead of concentrating on documented reports and observational results [27]. Eye tracking, sometimes referred to as eye-gaze tracking, provides better results than discount usability testing, as it produces both qualitative and quantitative data [28]. In this study, interactive eye tracking in which the states of the eye movement are recorded during an eye-tracking session, was used [27]. A saccade is a rapid, uneven progression of the eye as it changes attention between different points, and fixation is when the eye movement stops, when the eye captures needed information [29]. Examination of saccades delivers unbiased data about the thinking of participants while they are using [29], for example the Unisa and myUnisa websites.

\section{METHODOLOGY AND DATA COLLECTION}

\subsection{Population and Sampling}

The data in this paper was produced from research conducted among students with disabilities at Unisa. Usability evaluation of websites can be done with 5 to 20 participants [30-31]. This evaluation with 20 participants can be very expensive. The sample for the study consisted of 15 students with disabilities. There were 5 students in each group with differing challenges, such as limited hand functioning, visual and auditory disabilities. This research is based on the observation of the participants who interacted with the Unisa website and its e-learning website (myUnisa), based on their specific characteristics, in this instance, their disabilities. The sample for the study was obtained through purposive sampling which concentrates on people with particular experiences or qualities who will be able to assist in achieving the applicable research objectives.

\subsection{Data Collection Instrument}

The data was collected by eye tracking and observation while participants performed tasks in the controlled usability laboratory at Unisa. Ethical clearance for the project was obtained from Unisa. Students were informed that participation was voluntary and no rewards for participating in the study were made.

Fifteen volunteers (all Unisa students) with mobility (limited hand functioning), visual (partially sighted and low vision) and auditory disabilities were recruited to participate in this study. Each participant was given 20 minutes to interact with the website.

Only one participant at a time was tested in the usability laboratory in a controlled environment. The participants were put at ease, and told that the purpose of the usability test was to test the usability of the websites and not their ability in using the websites. They also signed the participant information sheets.

The video eye trackers were adjusted to each participant's eye actions through a calibration process in advance of the real testing in the usability laboratory [32]. All the participants had to follow a dot that appeared on the computer screen so that the centre of the pupil and reflection link of the cornea could be recorded by the system [32].

The participants were then requested to log on to or access the Unisa website (www.unisa.ac.za) to execute the tasks for the data collection as described below.
- $\quad$ Task 1

Use the mouse to discover complementary text to the images on the Unisa website homepage (move over the images with the mouse pointer - any image). Read the text and determine whether it explains the meaning of the images.

Motivation: User agents, which are the tools that individuals use to interact with electronic data, for example web browsers [31], are used to help people with disabilities to use alternative text to interpret images. Images must have alternative text which is descriptive enough, so that participants can understand the actual meaning of the pictures.

- $\quad$ Task 2

Use the main Unisa website www.unisa.ac.za to find a link to the myUnisa website.

Motivation: This task tested whether the language used in the website was natural and explanatory for participants to find other links.

- $\quad$ Task 3

Navigate from the homepage of Unisa website to a link of one more website page (other than the myUnisa website), such as Twitter, with ease.

Motivation: This was to determine whether participants were able to move from one page to another with ease and whether that page was the actual page the participant wanted to navigate to.

- $\quad$ Task 4

Log on to myUnisa (Unisa's e-learning website) in order to get information, for example the release date of exam results.

Motivation: This was to check if participants actually found the information they wanted.

During the eye-tracking sessions, the researcher observed participants' actions reflecting their experiences of using the Unisa and myUnisa websites.

\section{RESULTS AND DISCUSSION}

\subsection{Usability results and observation of participants in the usability laboratory}

A score to represent the level of difficulty experienced while performing the task was assigned to each of the tasks which participants performed. The scores used to reflect the complexity of the tasks are shown in Table 1.

Task 4 was dependent on the completion of Task 2. If a participant could not complete Task 2, Task 4 was denoted as 'not applicable'.

Table 1: Values assigned to reflect the complexity of tasks

\begin{tabular}{|l|c|}
\hline CONDITION & $\begin{array}{c}\text { VALUE } \\
\text { ASSIGNED }\end{array}$ \\
\hline Not applicable. & 9 \\
\hline No success in completing task. & 0 \\
\hline Severe difficulty but succeeded eventually with extra time. & 2 \\
\hline $\begin{array}{l}\text { Severe difficulty but, eventually succeeded within the time } \\
\text { limit, that is, 300 seconds (5 minutes). }\end{array}$ & 3 \\
\hline $\begin{array}{l}\text { Little to moderate difficulty, that is, 180 to 240 seconds (3 to } \\
4 \text { minutes). }\end{array}$ & 4 \\
\hline Easily, less than 180 seconds (3 minutes). & \\
\hline
\end{tabular}


The actual scores assigned to participants regarding how long they took to complete each task are recorded in Table 2.

Table 2: Time taken to complete each task with corresponding numerical values as per Table 1 for individual students with disabilities

\begin{tabular}{|l|l|l|l|l|c|}
\hline Task & $\begin{array}{l}\text { Task 1 } \\
\text { (Use the } \\
\text { mouse to } \\
\text { find } \\
\text { alternative } \\
\text { text) }\end{array}$ & $\begin{array}{l}\text { Task 2 } \\
\text { (Use the } \\
\text { main Unisa } \\
\text { website to } \\
\text { find a link } \\
\text { to the } \\
\text { myUnisa } \\
\text { website) }\end{array}$ & $\begin{array}{l}\text { Task 3 } \\
\text { (Navigate } \\
\text { from the } \\
\text { homepage } \\
\text { to a } \\
\text { webpage } \\
\text { other than } \\
\text { myUnisa) }\end{array}$ & $\begin{array}{l}\text { Task 4 } \\
\text { (Log on to } \\
\text { myUnisa and } \\
\text { get } \\
\text { information) }\end{array}$ & $\begin{array}{l}\text { Total } \\
\text { time } \\
\text { taken in } \\
\text { seconds }\end{array}$ \\
\hline 1 \\
\hline 2 & $2(252 \mathrm{sec})$ & $1(480 \mathrm{sec})$ & $3(240 \mathrm{sec})$ & $*$ & 972 \\
\hline 3 & $2(270 \mathrm{sec})$ & $2(276 \mathrm{sec})$ & $2(300 \mathrm{sec})$ & $2(258 \mathrm{sec})$ & 1104 \\
\hline 4 & $2(276 \mathrm{sec})$ & $1(396 \mathrm{sec})$ & $2(300 \mathrm{sec})$ & $2(288 \mathrm{sec})$ & 1260 \\
\hline 5 & $2(294 \mathrm{sec})$ & $1(420 \mathrm{sec})$ & $2(300 \mathrm{sec})$ & $3(192 \mathrm{sec})$ & 1206 \\
\hline 6 & $2(300 \mathrm{sec})$ & $2(276 \mathrm{sec})$ & $2(300 \mathrm{sec})$ & $2(264 \mathrm{sec})$ & 1140 \\
\hline 7 & $2(264 \mathrm{sec})$ & $1(432 \mathrm{sec})$ & $1(360 \mathrm{sec})$ & $*$ & 1056 \\
\hline 8 & $2(252 \mathrm{sec})$ & $1(360 \mathrm{sec})$ & $2(270 \mathrm{sec})$ & $*$ & 882 \\
\hline 9 & $2(246 \mathrm{sec})$ & $1(480 \mathrm{sec})$ & $2(300 \mathrm{sec})$ & $*$ & 1026 \\
\hline 10 & $2(270 \mathrm{sec})$ & $2(258 \mathrm{sec})$ & $2(300 \mathrm{sec})$ & $2(282 \mathrm{sec})$ & 1110 \\
\hline 11 & $2(282 \mathrm{sec})$ & $1(420 \mathrm{sec})$ & $2(300 \mathrm{sec})$ & $*$ & 1002 \\
\hline 12 & $3(228 \mathrm{sec})$ & $3(210 \mathrm{sec})$ & $4(66 \mathrm{sec})$ & $3(216 \mathrm{sec})$ & 720 \\
\hline 13 & $3(210 \mathrm{sec})$ & $1(360 \mathrm{sec})$ & $4(60 \mathrm{sec})$ & $*$ & 630 \\
\hline 14 & $4(120 \mathrm{sec})$ & $3(192 \mathrm{sec})$ & $4(90 \mathrm{sec})$ & $3(186 \mathrm{sec})$ & 588 \\
\hline 15 & $4(156 \mathrm{sec})$ & $3(234 \mathrm{sec})$ & $4(60 \mathrm{sec})$ & $3(198 \mathrm{sec})$ & 648 \\
\hline
\end{tabular}

The tasks with the completion times by students with disabilities, to show how long it took each participant to perform each task, are tabulated in Table 2 .

In Table 2, it must also be noted that, in order to do Task 4 , participants first had to do Task 2. If the participants could not do Task 2, then Task 4 was not applicable. In Table 2 above, it can be seen that participants 1 to 5 had limited hand functioning, 6 to 10 had visual disabilities and 11 to 15 had auditory disabilities. Even though total time taken to complete tasks for participants $6,7,8$ and 10 was less than 1200 seconds, they did not complete Task 4 and they took a long time to complete Task 2.

Table 3 represents usability principles not satisfied by the Unisa and myUnisa websites, as per observation by the researcher based on the performance of participants with disabilities in the usability laboratory.

Table 3: Usability principles not satisfied by Unisa and myUnisa websites

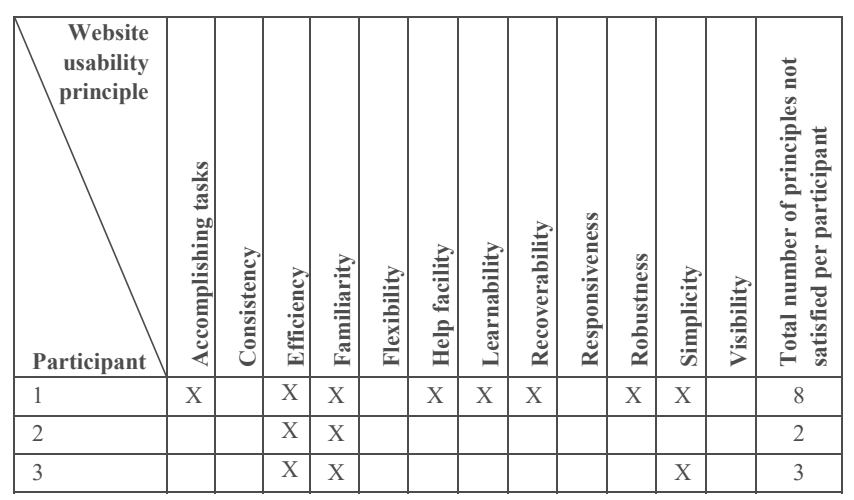

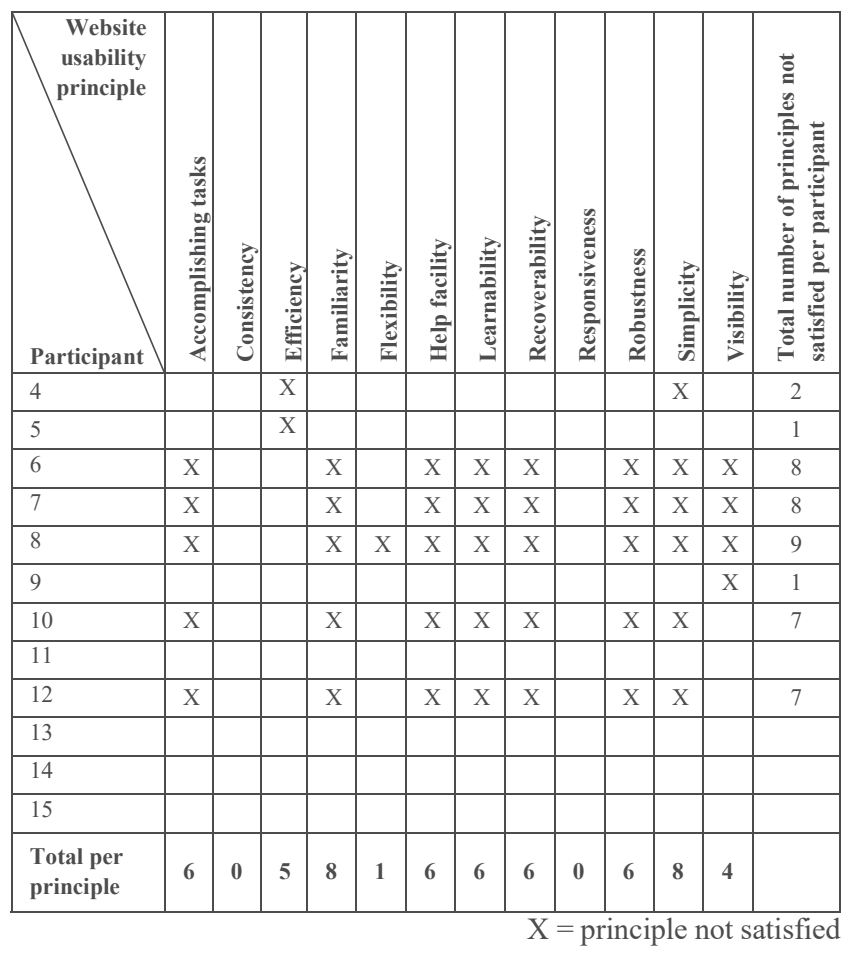

The principles that were not satisfied are as follows (see Table 3 above):

- Accomplishing tasks - the Unisa and myUnisa websites did not cater for 6 of the 15 participants with disabilities, that is $40 \%$.

- Efficiency - the Unisa and myUnisa websites did not satisfy 5 of the 15 participants with disabilities, that is $33.3 \%$.

- Familiarity - the Unisa and myUnisa websites did not cater for 8 of the 15 participants with disabilities, that is $53.3 \%$.

- Flexibility - the Unisa and myUnisa websites did not satisfy 1 of the 15 participants with disabilities, that is $6.7 \%$.

- Help facility - the Unisa and myUnisa websites did not satisfy 6 of the 15 participants with disabilities, that is $40 \%$.

- Learnability - the Unisa and myUnisa websites did not satisfy 3 of the 15 participants with disabilities, that is $20.0 \%$.

- Recoverability - the Unisa and myUnisa websites did not cater for 6 of the 15 participants with disabilities, that is $40 \%$ across all the disabilities.

- Robustness - the Unisa and myUnisa websites did not satisfy 6 of the 15 participants with disabilities, that is $40 \%$.

- $\quad$ Simplicity - the Unisa and myUnisa websites did not satisfy 8 of the 15 participants with disabilities, that is 53.3 . 
- Visibility - the Unisa and myUnisa websites did not satisfy 4 of the 15 participants with disabilities, that is $26.6 \%$.

\subsection{Further discussion of usability results and observations}

A quick glance at the results in Table 3 reveals that the usability principles which were best satisfied are consistency and responsiveness, while familiarity and simplicity of the Unisa and myUnisa websites were the most problematic principles for these participants.

From Table 2 it can be seen that participants 1, 6, 7, 8, 10 and 12 did not finish Task 4. Four of the participants who did not finish their tasks had visual disabilities. Extra time was allowed for these participants to do Task 2. It can also be seen that participants 3 and 4 did not finish tasks within the allotted time of 1200 seconds ( 20 minutes). Given the fact that it was difficult to finish tasks and that some participants did not complete tasks in the allotted time, we conclude that the usability principles of familiarity and simplicity were not satisfied by the Unisa and myUnisa websites.

Tables 2 and 3 seem to suggest that the participants with auditory disabilities performed much better than those with limited hand functioning and visual disabilities. All participants with limited hand functioning disabilities, that is, participants 1 to 5 , were less efficient when doing their tasks (see Tables 2 and 3). They took much longer than other participants to do the tasks.

For participants with visual disabilities, the usability principles not satisfied by the Unisa and myUnisa websites include accomplishing tasks, familiarity, help facility, learnability, recoverability, robustness, simplicity and visibility. As seen in Table 3, the total for principles not satisfied was much higher for these participants than for limited hand functioning and auditory disability participants.

Participants with limited hand functioning and visual disabilities performed tasks in more or less the same amount of time, that is, participants 1 to 5 and 6 to 10 , as seen in Table 3 .

There were issues with familiarity and simplicity of the Unisa and myUnisa websites for 3 participants with limited hand functioning, 4 participants with visual disabilities and 1 participant with an auditory disability. The familiarity and simplicity principles were the principles of usability least satisfied by the Unisa and myUnisa websites, with $53.3 \%$ each. Participants had little or no training in the use of the Unisa and myUnisa websites. Specific problems that students with disabilities, especially those with limited hand functioning and visual disabilities, experienced on the Unisa and myUnisa websites, as identified during the controlled usability evaluation, were as follows:

- The participants were not able to use the websites without their respective assistive technologies. For example, students were not aware of an option to enlarge text on the Unisa website and possibly the myUnisa website.

- The participants struggled to get to the myUnisa link from the Unisa website.

- It was challenging for the participants to get to the right webpages.

- Some participants forgot their myUnisa passwords and could not find out how to retrieve or reset it on/from the myUnisa website, because it was not easy to find the link to change passwords.

- Participants took a long time to discover certain links from the Unisa website.

\section{CONCLUSIONS}

The objectives of this study were to examine how time of task completion relates to the usability principles, observe the principles which are problematic when participants accomplish specific tasks on the Unisa website and Unisa's e-learning website (myUnisa) and recommend ways to improve efficiency and effectiveness of the websites at this ODeL institution.

All the participants who were not able to do Task 2 and therefore also not Task 4 found that the Unisa and myUnisa websites were not simple to use or were difficult to use. These participants experienced difficulties in using these websites because they were not familiar with them. This means that the UDL principles, such as offering multiple means of representation, several ways of action and expression and multiple means of engagement with learning matters, were not satisfied.

The principles which were mostly not satisfied are familiarity and simplicity. These principles need attention as participants took a lot of time to accomplish tasks on the Unisa and myUnisa websites.

The quantitative model of usability was not satisfied as most of the participants did not finish tasks in time with few errors. It can be seen from Tables 2 and 3 that most of the usability principles were not satisfied by the Unisa and myUnisa websites for the participants with limited hand functioning and visual disabilities. Therefore the participants with these disabilities could not complete the tasks on time with no or minimal errors to the user's satisfaction. If the usability principles are implemented properly, they will contribute to usable websites. If websites are usable, then they are accessible. Websites should be developed in such a way that students with disabilities can achieve the results they envisage.

E-learning websites such as myUnisa are supposed to provide students with learning experiences and not only support with, for example, submission of assignments. There is a need for interactive and inclusive Unisa and myUnisa websites which will meet the educational needs of all students, including students with disabilities, so that digital and social divides and the marginalisation of students can be nullified or reduced. To improve the accomplishment of tasks, the institutional websites must be designed in such a way that all students can visibly recover from errors, and they need to at least be interactive so that people with disabilities will be able to use the technology properly. Training to use assistive technologies or to use the websites needs to be given to users. Universally designed technologies should also be incorporated in the Unisa and 
myUnisa websites to make these websites accessible to all students, regardless of their abilities and competencies. The Internet and computing facilities of this institution need to be improved to provide for the e-learning necessities of all students.

For the Unisa and myUnisa websites to cater for applicable disabilities of students, assistive technologies for each type of disability should be provided. It is worth noting that students with disabilities need to participate in the actual development or improvement of any websites which affect them. E-learning websites, in particular, must meet users' requirements when these users are tasked with using the technology for learning purposes. Students with disabilities should additionally be provided with ICT training to enhance their experiences in the use of websites in general as well as e-learning websites. Internet, broadband and computing facilities should be made available wherever students live, including students with disabilities and those who reside in rural areas. This will meet the needs of students with disabilities with regard to e-learning. E-learning websites must be developed specifically to simplify the learning process of students at this ODeL institution as students from all over the world study there.

In order to provide for all students, including those with disabilities, e-learning websites must allow different means of learning and multiple ways of solving problems to show their knowledge and properly engage students. This will encourage them to use the Unisa and myUnisa websites on a regular basis.

\section{ACKNOWLEDGMENTS}

Thank you to all participants who assisted with the collection of data.

\section{REFERENCES}

[1] Nicolle, C. and Abascal, J. 2001. Inclusive design guidelines for HCI. Boca Raton, FL: CRC Press.

[2] Munro, M. and McMullin, B. 2009. E-Learning for all? Maximizing the impact of multimedia resources for learners with disabilities, used e-learning and e-teaching in higher education. London: Information Science Reference.

[3] Abou-Zahra, S. 2012. How people with disabilities use the Web: W3C working draft [online]. Available from: $<$ http://www.w3.org/WAI/intro/people-use-web/> [Accessed 9 June 2012].

[4] Horton, S. and Leventhal, L. 2008. 'Universal usability'. In: Harper, S. and Yesilada, Y. Eds. Web accessibility: A foundation for research. 345-355.

[5] Lidwell, W., Holden, K. and Butler, J. 2010. Universal principles of design: 125 ways to enhance usability, influence perception, increase appeal, make better design decisions, and teach through design. Gloucester, MA: Rockport Pub.

[6] Sah, M. and Wade, V. 2010. Automatic metadata extraction from multilingual enterprise content: Proceedings of the 19th ACM International Conference on Information and Knowledge Management, Toronto, Canada, October 2630, 2010. ACM.

[7] United Nations (UN). 2006. Convention on the Rights of Persons with Disabilities [online]. Available from: $<\mathrm{http} / / / \mathrm{www}$. un.org/disabilities/default. asp?id=259> [Accessed 14 March 2017].

[8] Center for Applied Special Technology (CAST). 2011. Universal design for

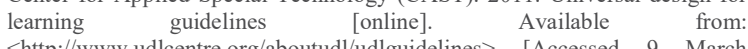
$<$ http://www.udlcentre.org/aboutudl/udlguidelines $>$ [Accessed 9 March 2012].

[9] Center for Applied Special Technology (CAST). 2010. UDL questions and answers [online]. Available from: <http://www.cast.org/udl/faq/index.html> [Accessed 9 March 2017].

[10] International Organization for Standardization (ISO). 2008. Ergonomics of human-system interaction: Guidance on software accessibility. Geneva.

[11] Slatin, J.M. and Rush, S. 2003. Maximum accessibility: Making your website more usable for everyone. Boston, MA, USA: Addison-Wesley Professional.

[12] Preece, J., Rogers, Y. and Sharp, H. 2011. Interaction design: Beyond human- computer interaction. Hoboken, NJ: Wiley.

[13] Kuakiatwong, S. 2012. Evaluating web accessibility and usability for totally blind users at Thailand Cyber University. Boston: Boston University Press.

[14] Bevan, N., Carter, J and Harker, S. 2015, August. ISO 9241-11 revised: What have we learnt about usability since 1998? In International Conference on Human-Computer Interaction (pp. 143-151). Springer, Cham.

[15] Nielsen, J. 1993. Usability engineering. Boston: Academic Press.

[16] Sauro, J. and Kindlund, E. 2005. A method to standardize usability metrics into a single score. In Proceedings of the SIGCHI Conference on Human Factors in Computing Systems (pp. 401-409). ACM.

[17] Moore, M., Bias, R.G., Prentice, K., Fletcher, R. and Vaughn, T. 2009. 'Web usability testing with a Hispanic medically underserved population', Journal of the Medical Library Association, 97(2): 114 - 121.

[18] Dumas, J.S. and Fox, J.E. 2008. Usability testing: Current practice and future directions. In: Sears, A. and Jacko, J.A. eds. The human-computer interaction handbook: fundamentals, evolving technologies, and emerging applications. New York: Lawrence Erlbaum Associates. 232-249.

[19] Budd, A. 17 January 2007. Heuristics for modern web application development. [Blog entry] Available from: $<$ www.andybudd.com/archives $/ 2007 / 01 /$ heuristics_for_modern_web_develop ment $>$ [Accessed 12 February 2014].

[20] Dix, A.J., Finlay J., Abowd G. and Beale R. 2004. Human-computer interaction. New York: Pearson/Prentice Hall.

[21] Mayhew, D.J. 1991. Principles and guidelines in software user interface design. Englewood Cliffs, NJ: Prentice-Hall, Inc.

[22] Molich, R. and Nielsen, J. 1990. 'Improving a human-computer dialogue', Communications of the ACM, 33(3): 338-348.

[23] Norman, D.A. 2001. The design of everyday things. London: MIT Press.

[24] Petrie, H. and Power, C. 2012. What do users really care about? A comparison of usability problems found by users and experts on highly interactive websites: Proceedings of the 2012 ACM Annual Conference on Human Factors in Computing Systems, Austin, Texas, May 5-10, 2012. ACM.

[25] Shneiderman, B. and Plaisant, C. 2010. Designing the user interface: Strategies for effective human-computer interaction. New Jersey: AddisonWesley.

[26] Tognazzini, B. 2003. First principles of interaction design, interaction design solutions [online]. Available from: <http://asktog.com/atc/principles-ofinteraction-design/ $>$ [Accessed 12 May 2014].

[27] Kules, B. and Capra, R. 2012. 'Influence of training and stage of search on gaze behavior in a library catalog faceted search interface', Journal of the American Society for Information Science and Technology, 63(1): 114-138.

[28] Alt, F., Shirazi, A.S., Schmidt, A. and Mennenöh, J. 2012. Increasing the user's attention on the web: Using implicit interaction based on gaze behavior to tailor content: Proceedings of the 7th Nordic Conference on Human- Computer Interaction: Making Sense Through Design, Copenhagen, October 14-17, 2012. ACM.

[29] Penzo, M. 2005. Introduction to eye tracking: Seeing through your users eyes [online]. Available from: $<$ http://uxmatters.com/MT/archives/000040.php.> [Accessed 9 June 2012].

[30] Nielsen, J. 2006. Quantitative studies: How many users to test. Alertbox [online]. Available from: http://www.useit.com/alertbox/quantitative_testing.html. [Accessed 14 July 2012].

[31] Nielsen, J. 2000. Why you only need to test with 5 users. Jakob Nielsen's Alertbox [online]. Available from: http://www.useit.com/alertbox/20000319.html. [Accessed 14 July 2012].

[32] Poole, A. and Ball, L. J. 2006. 'Eye tracking in HCI and usability research', Encyclopedia of Human Computer Interaction, 1: 211-219. 